\title{
METALOSIS POR TITANIO. DIAGNOSTICO DIFERENCIAL DE MASA DE COMPARTIMIENTO ILIOPSOAS. CASO CLINICO
}

Int. Evelyn May $\mathrm{G}^{(1)}$, Drs. Andrés O’Brien $\mathrm{S}^{(2)}$, Carlos Liendo ${ }^{(3)}$, Cristián Ortiz $\mathbf{M}^{(3)}$, Int. Claudia López $\mathrm{D}^{(1)}$.

1. Medicina. Pontificia Universidad Católica de Chile.

2. Departamento de Radiología. Pontificia Universidad Católica de Chile.

3. Departamento de Traumatología Pontificia Universidad Católica de Chile.

\begin{abstract}
An 52 year-old female patient with right hip joint Tuberculosis, received a Titanium total joint arthroplasty in 1991. Eleven years later, she presented low extremity swelling and inguinal mass, which was studied with ultrasound and computed tomography. The main differential diagnosis was an iliopsoas sarcoma, so an ultrasound-guided biopsy was performed. The result was Titanium particle debris. The lesion was removed, but it recurred, so that joint replacement was required.

Joint Titanium particle wear debris a condition due to particle releaseis as an arthroplasty complication. When its clinical manifestation is a pelvic mass, the main differential diagnoses include tumors and granulomatous disease. Both clinical manifestations and the radiology are variable. This pathology in patient with hip joint Titanium arthroplasty must be consided.
\end{abstract}

Key words: Hip joint arthroplasty, Particle wear debris, Pelvic mass, Titanium prosthesis.

Resumen: Se presenta el caso clínico de una paciente de 52 años con diagnóstico de tuberculosis de cadera derecha a quien en 1991 se le instala prótesis de Titanio. Once años después presenta aumento de volumen de la extremidad inferior y masa palpable en zona inguinal. Fue estudiada con ultrasonografía y tomografía computada cuyos hallazgos se describen. Se planteó como diagnóstico diferencial principal un sarcoma en compartimiento iliopsoas por lo cual se realiza biopsia bajo ultrasonografía que demostró metalosis por Titanio diagnóstico que debe ser considerado en pacientes con masa inguinal y prótesis de cadera. Se re-

May E. y cols. Metalosis por titanio. Diagnóstico diferencial de masa de compartimiento iliopsoas. Caso clinico. Rev Chil Radiol 2002; 8: 182-184.

Correspondencia: Int. Evelyn Milay G. Hospital Clínico Pontificia Universidad Católica de Chile. Departamento de Radiología. Marcoleta $N^{\circ}$ 367, 2ำ piso. Santiago. Chile. EMail:emay@puc.cl seca la lesión, la cual recidiva, requiriendo recambio articular.

Palabras claves: Artroplastía total de cadera, Masa pélvica, Metalosis, Prótesis de Titanio.

\section{Introducción}

La metalosis por Titanio es una patología que se produce por liberación de partículas de este material como complicación de una artroplastía. Los diagnóstico diferenciales principales cuando se presenta como masa pélvica incluyen tumores y patología granulomatosa. Su presentación clínica es variable, al igual que las manifestaciones radiológicas. Dada la importancia de considerar esta patología en pacientes con prótesis de cadera y masa en región inguinal se presenta un caso con comprobación histológica.

\section{Caso clínico}

Paciente del sexo femenino de 52 años a quien se le diagnóstica TBC de cadera derecha en 1975, motivo por el cual el año 1991 se efectuó artroplastía total de cadera (ATC), instalándose prótesis de Titanio (Figura 1).

Después de once años, la paciente consulta por aumento de volumen de la extremidad inferior derecha asociado a masa palpable en fosa ilíaca ipsilateral que se estudia mediante ultrasonografía (Figura 2) y tomografía computada (TC) (Figura 3). Se informó una masa sólido-quística del compartimiento iliopsoas, cuyo diagnóstico diferencial incluía como primera posibilidad una patología tumoral. Se plantean dentro de los diagnósticos diferenciales un probable sarcoma, y menos probablemente una patología inflamatoria.

Se efectúa biopsia guiada por ultrasonografía de la masa pelviana, cuyo resultado fue metalosis por Titanio. Se realiza resección quirúrgica de la lesión cuyo estudio histológico y el de algunos ganglios inguinales resecados confirmaron el diagnostico (Figura 4). La paciente se mantiene asintomática por aproximadamente 5 meses luego de los cuales re- 
aparece la masa en fosa ilíaca derecha. Se repite estudio con TC (Figura 5), el que muestra similares hallazgos a los previamente descritos. Dada esta situación, se decide realizar recambio articular.

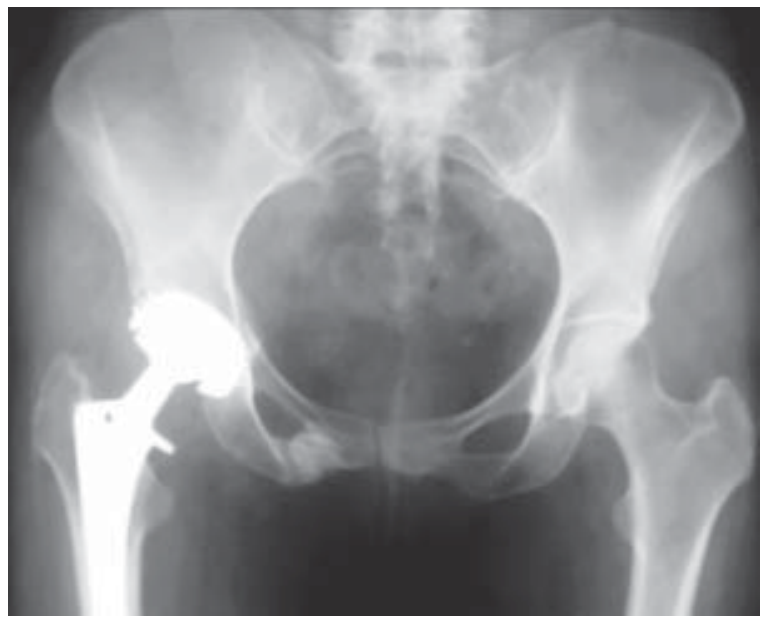

Figura 1. Radiografía AP de pelvis que muestra artroplastía total de cadera derecha.
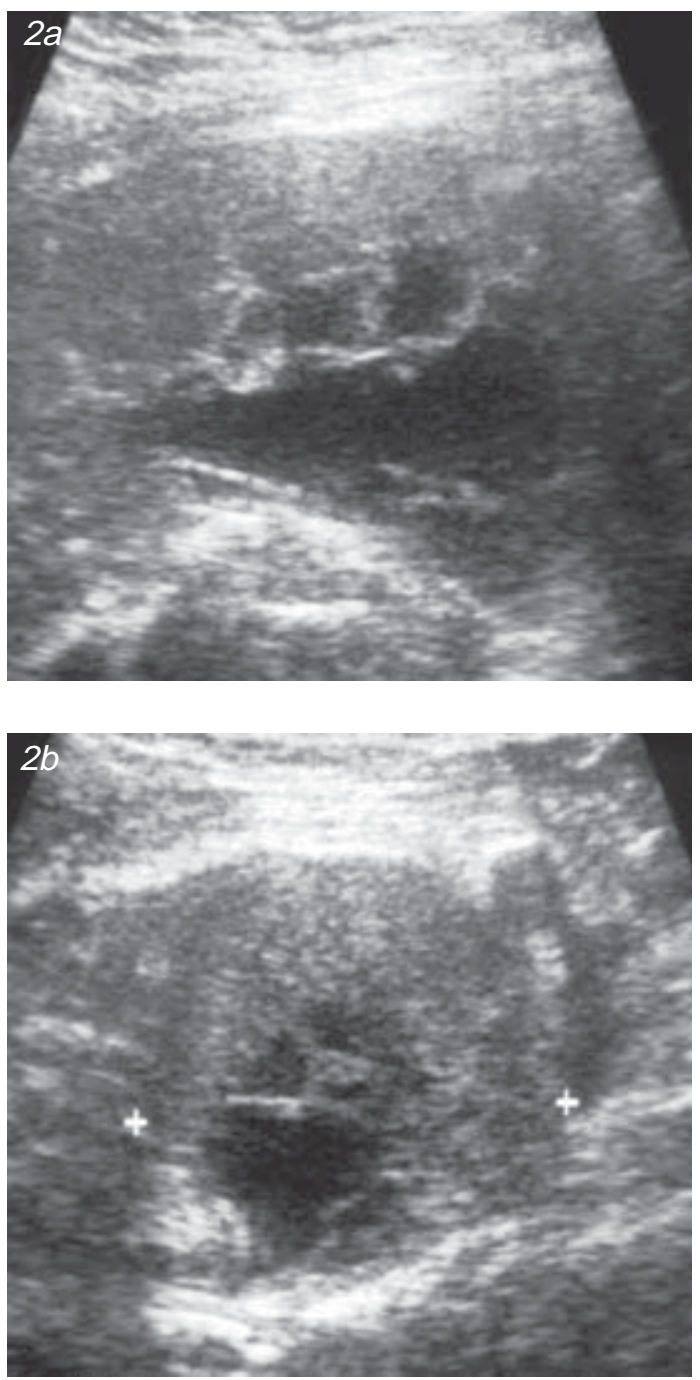

Figura 2 a,b. Ecografía corte longitudinales en la que se demuestra masa sólido-quística que medía aproximadamente $8 \mathrm{~cm}$ de diámetro mayor.

\section{Discusión}

La ATC ha contribuido en forma importante al alivio de pacientes con distintas patologías; no obstante, la existencia de elementos que llevan a la falla de ésta ha constituido un problema importante en las últimas décadas.

Los mecanismos de falla de una ATC se pueden dividir en infecciosos y no infecciosos. Dentro de estos últimos se encuentra el que afectó a nuestra paciente, la falla por desgaste del implante, que corresponde a pérdida de material con generación de partículas, en este caso de la aleación de Titanio, lo que se asocia a un extenso proceso inflamatorio ${ }^{(1,2,4,6,9-11)}$.
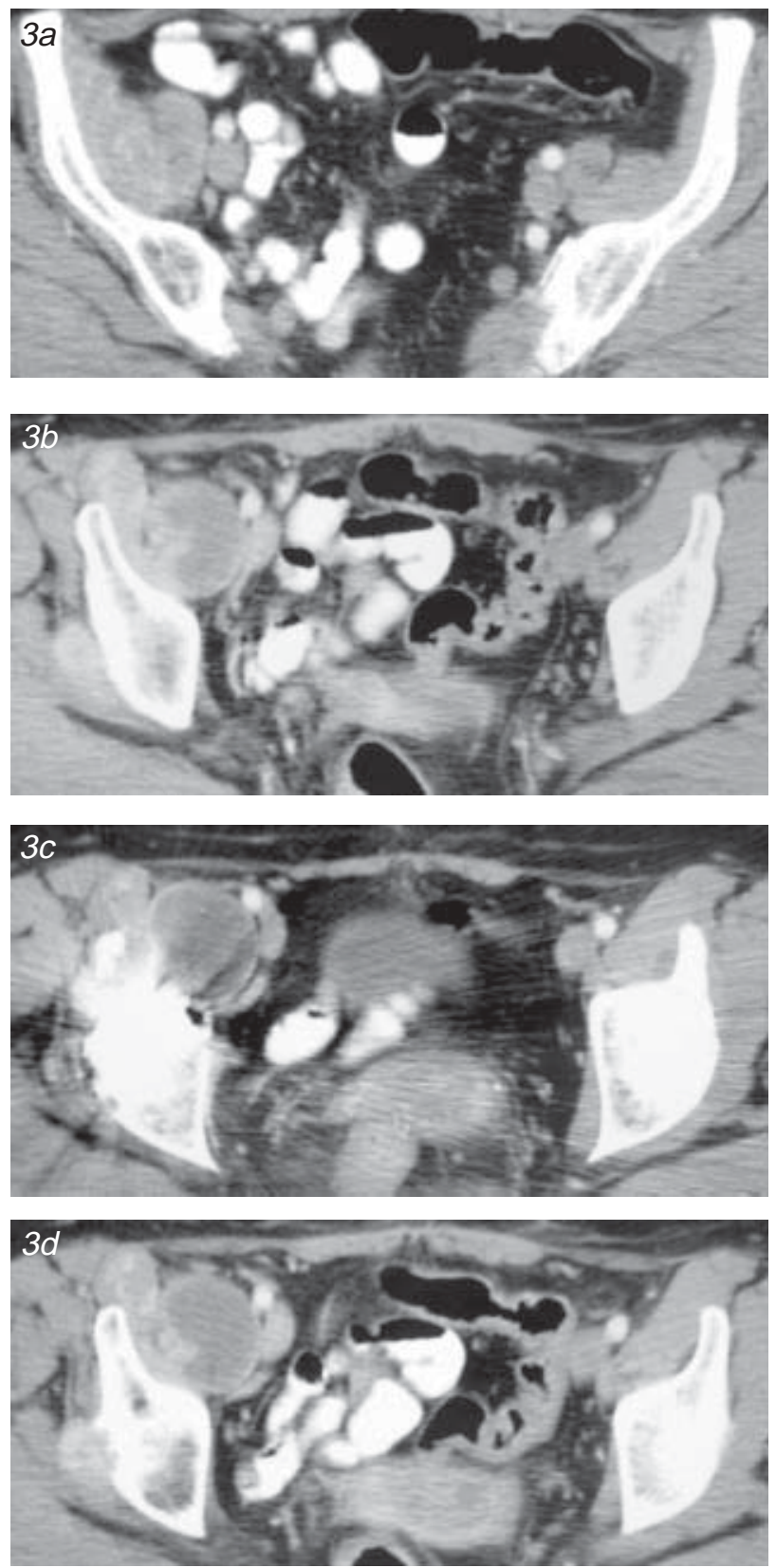

Figura $3 \mathbf{a}, \boldsymbol{b}, \boldsymbol{c}, \boldsymbol{d}$. TAC a nivel de pelvis demostrando masa heterogénea con áreas hipodensas en el compartimiento de iliopsoas derecho en estrecha relación con la prótesis como se demuestra en el corte inferior (c). 

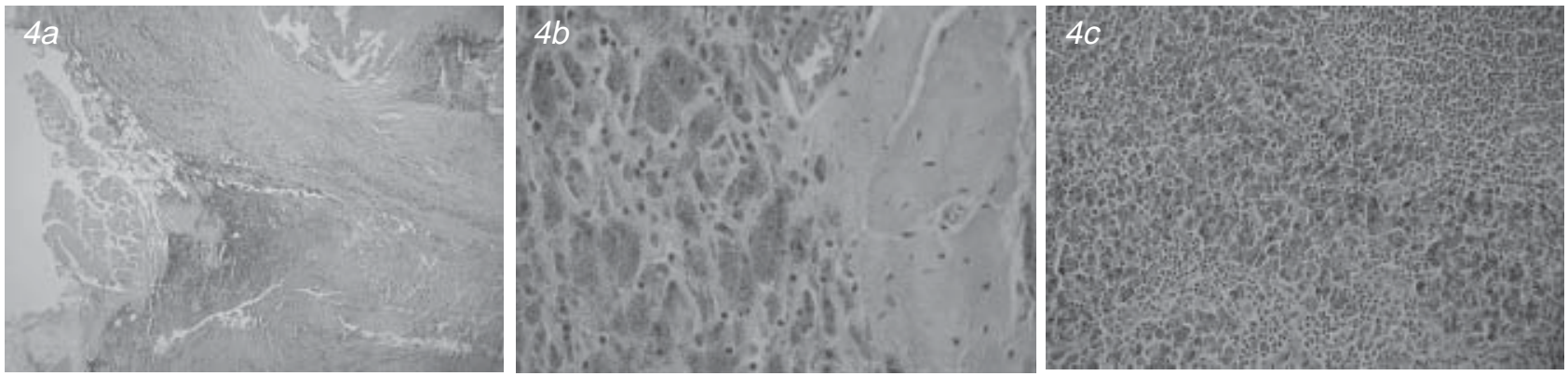

Figura 4 a,b,c. (a) Muestra de masa pélvica con tinción de Hematoxilina-Eosina (H-E) a bajo aumento. Se observan bandas de tejido conectivo organizado, entre las que se identifican abundantes fibroblastos con contenido granular pardo-negruzco en su citoplasma. En la periferia hay algunos haces musculares y tejido adiposo. (b) Muestra de masa pélvica con tinción de H-E a gran aumento. Se identifican con claridad partículas granulares pardo-negruzcas al interior de fibroblastos y entre las fibras de tejido conectivo. Algunas de estas células no contienen el material particulado. (c) Muestra de adenopatía inguinal con tinción de H-E a mediano aumento. Se observan abundantes células histiocitarias con contenido granular pardo-negruzco en su citoplasma. Alrededor hay células linfáticas.
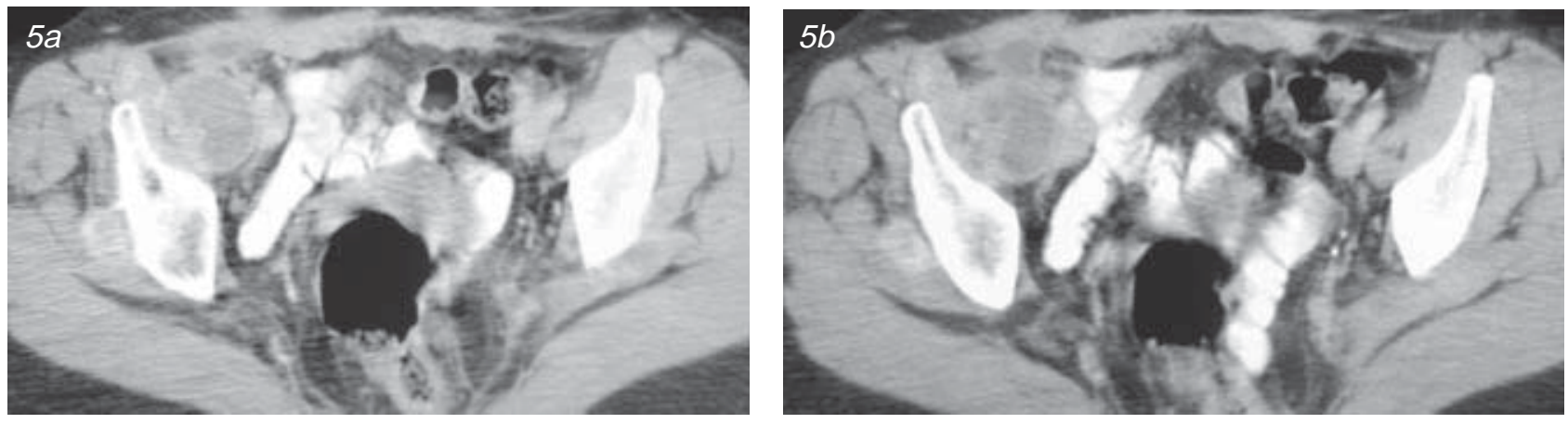

Figura 5. TAC 5 meses post biopsia. Se demuestra recidiva de la lesión que compromete el iliopsoas derecho.

La aleación de Titanio (Titanio-Aluminio-Vanadio), posee una serie de ventajas en relación a su utilización, destacando su gran biocompatibilidad ${ }^{(9)}$. Sin embargo, existen también desventajas, en las cuales se incluyen la mayor generación de partículas y una menor tolerancia al desgaste, comparado con otros tipos de aleaciones ${ }^{(3,7-9)}$.

La presentación clínica de una ATC complicada con liberación de partículas puede ser muy variada, pudiendo constituir un hallazgo imagenológico y en otras ocasiones manifestarse como una diseminación sistémica de

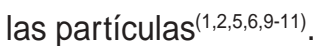

Existen distintas manifestaciones imagenológicas de esta patología, que dependerán, tanto del tipo de examen realizado como de la presentación clínica. Los diagnósticos diferenciales más comunes son tumores pélvicos, principalmente sarcomas, enfermedades granulomatosas, etc.

En general, en la radiografía simple la manifestación más común es la presencia de zonas lobuladas de osteolisis periprotésica, con o sin aumento de densidad de partes blandas adyacentes. En la tomografía computada pueden apreciarse alteraciones óseas más la presencia de masa periprotésica, generalmente hipodensa. La resonancia magnética puede mostrar masas focales intraóseas hipointensas en T1 y con intensidad de señal intermedia o baja, heterogéneas en $T 2^{(12)}$.

En conclusión, es importante considerar metalosis en el diagnóstico diferencial de masa en compartimiento iliopsoas, en pacientes con prótesis de Titanio.

\section{Bibliografía}

1. Bauer T, Schils J. The pathology of total joint arthroplasty. II: Mechanisms of implant failure. Skeletal Radiol 1999; 28: 483-497.

2. Benz E, Sherburne B, Hayek, J. et al. Lymphadenopathy associated with total joint prostheses. A report of two cases and a review of the literature. J Bone Joint Surg 1996; 78: 479-481.

3. Black J, Sherk H, Bonini J. et al. Metallosis associated with a stable titanium-alloy femoral component in total hip replacement. J Bone Joint Surg 1990; 72: 126.

4. Glant T, Jacobs J, Mikecz K, et al. Particulate-induced, prostaglandinand cytokine-mediated bone resorption in an experimental system and in failed joint replacements. Am J Ther 1996; 3:27-41.

5. Hattrup S, Bryan R, Gaffey T, Stanhope C. Pelvic mass causing vesical compression after total hip arthroplasty. Case Report Clin Orthop 1988; 227: 184-189.

6. Lombardi A, Mallory T, Vaughn B, Drovillard P. Aseptic loosening in total hip arthroplasty secondary to alloy modular femoral heads. J Bone Joint Surg Am 1989; 71: 1337-1342.

7. Maloney W, Smith L, Schmalzried T, et al. Isolation and characterization of wear particles generated in patients who have had failure of a hip arthroplasty without cement. J Bone Joint Surg Am 1995; 77: 13011310.

8. Mirra J, Marder R, Amstutz $\mathrm{H}$. The pathology of failed total joint arthroplasty. Clin Orthop 1982; 170: 175-183.

9. Salvati E, Betts F, Doty S. Particulate metallic debris in cemented total hip arthroplasty. Clin Orthop 1993; 293: 160-173.

10. Urban R, Jacobs J, Tomlinson M, et al. Dissemination of wear particles to the liver, spleen, and abdominal lymph nodes of patients with hip or knee replacement. J Bone Joint Surg Am 2000; 82: 457-476.

11. Wang J, Lin C. Pelvic mass caused by polyethylene wear after uncemented total hip arthroplasty. J Arthroplasty 1996; 11:626-628.

13. White LM, Kim J, Merchant M, et al. Complications of total hip arthroplasty: MR imaging initial experience. Radiology 2000;215:254-62. 